Ann. Biol. anim. Bioch. Biophys., 1979, 19 (2 A), 337-341.

\title{
Colostrum de vache : composition minérale et activité de la phosphatase alcaline
}

\author{
par G. LINDEN, B. MARAVAL * \\ Laboratoire de Biochimie Appliquée. Université de Nancy I, Cose officielle $n^{\circ}$ 140, 54037 Nancy Cedex. \\ * Laboratoire de Zootechnie-E. N. S. A. I. A., 38, rue Ste Catherine, 54000 Nancy.
}

Summary. Cow colostrum. Mineral composition and alkaline phosphatase activity.

The correlation pattern between the quantities of alkaline phosphatase and those of zinc, calcium, magnesium and phosphorus released in the milk during the colostrumproducing period was always positive and the coefficients often high. These results suggested a very close localization of the alkaline phosphatase and the bivalent ions and indicated a possible participation of the enzyme in the transport of those substances.

\section{Introduction.}

Les teneurs en éléments minéraux du lait de vache ont fait l'objet d'innombrables travaux. L'étude des variations de la composition minérale du lait en fonction du stade de lactation a permis de montrer qu'en règle générale, le colostrum de vache est plus riche en éléments minéraux que le lait normal (Guéguen, 1971 ; Maraval et Vignon, 1977).

La phosphatase alcaline du laif sous sa fraction dominante est un dimère de 170000 daltons qui comprend 4 atomes-g de zinc par mole de protéine et qui requiert deuxtypes de métaux pour une activité maximale : le zinc qui est essentiel ef le magnésium qui est activateur. D'aułres cations bivalents peuvent également stimuler son activité (Linden et Alais, 1976 ; Linden et al., 1977).

Nous avons examiné en parallèle dans le colostrum les variations des teneurs en calcium, magnésium, sodium, potassium, phosphore, zinc et en phosphatase alcaline.

\section{Matériel et méthodes.}

Les mesures ont été effectuées sur les laits de 14 animaux. 6 vaches sont de race Française Frisonne Pie Noire (FFPN) et 8 vaches sont issues du croisement FFPNHolstein.

Pendant la période expérimentale, les vaches ont reçu des aliments concentrés : 6 vaches ont reçu une ration composée en moyenne de $8,7 \mathrm{~kg}$ de matière sèche d'ensilage de maīs et $3,9 \mathrm{~kg}$ de matière sèche pâturée ; les 8 autres animaux ont eu un 
régime composé uniquement de $12,6 \mathrm{~kg}$ de matière sèche d'ensilage de maïs. Un échantillon de chacune des 8 premières traites de chaque animal a été prélevé et analysé. Les colostrums ont été additionnés, après prélèvement, de 2 gouttes de toluène puis congelés. Les 8 traites d'une vache ont été analysées le même jour.

La mesure de l'activité phosphatasique a été effectuée par la méthode d'Aschaffenburg et Mullen (1948) modifiée. L'activité phosphatasique est exprimée en quantité de p-nitrophénol libéré à $37^{\circ} \mathrm{C}$ par minute et par kilogramme de lait. La répétabilité de la méthode est bonne, car, en dosant dix-huit fois de suite l'activité du même lait, on obtient : $513 \pm 5,9 \mu$ moles. $\mathrm{mn}^{-1} \cdot \mathrm{kg}^{-1}$.

Les dosages du calcium et magnésium ont été effectués par spectrométrie d'absorption atomique et ceux du sodium et potassium par spectrométrie d'émission de flamme selon les protocoles décrits précédemment (Linden et al., 1970). La détermination de la teneur en zinc a également été effectuée par spectrométrie d'absorption atomique selon la méthode décrite par Bellanger et Lamand (1975). Le dosage du phosphore a été réalisé selon la technique de Bamman et al. (1948).

Sur les résultats des différentes analyses, nous avons calculé les moyennes et écarts-types. Les comparaisons de moyennes ont été effectuées par l'analyse de variance de Fisher et par le test de Duncan. Nous avons également calculé les coefficients de corrélation entre les quantités de phosphatase alcaline et les quantités d'éléments minéraux excrétées dans le lait.
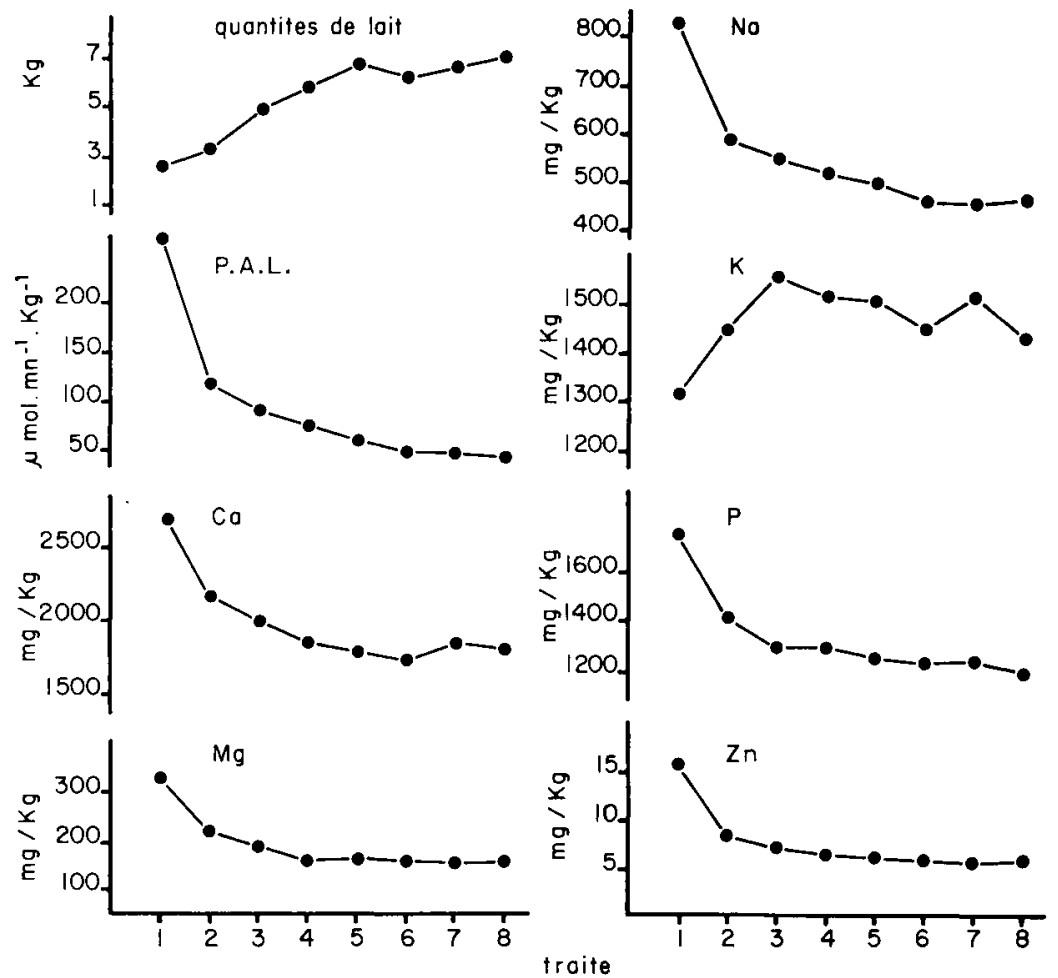

FIG. 1. - Evolution des quantités de lait produites, des teneurs en phosphatase alcaline (P. A. L.) ef des feneurs en $\mathrm{Ca}, \mathrm{Mg}, \mathrm{Na}, \mathrm{K}, \mathrm{P}$ ef $\mathrm{Zn}$ au cours des huit premières traites. 


\section{Résultats.}

Pour l'ensemble des animaux, les différences entre les quantités de lait produites au cours des 8 premières traites qui suivent la mise bas sont hautement significatives. Les quantités de lait produites augmentent rapidement entre la $1 \mathrm{re}$ traite $(2,54 \mathrm{~kg})$ et la $3 \mathrm{e}$ traite $(5,07 \mathrm{~kg})$ puis plus lentement ensuite $(7,00 \mathrm{~kg}$ à la $8 \mathrm{e}$ traite) (fig. 1$)$.

Au cours de cette phase colostrale, l'effet du numéro de traite est toujours hautement significatif pour les teneurs en phosphatase alcaline, zinc, calcium, magnésium, sodium et phosphore. Les teneurs en ces différents éléments minéraux diminuent de façon importante entre la 1 re traite $(\mathrm{Zn}: 15 \mathrm{mg} / \mathrm{kg}, \mathrm{Ca}: 2571 \mathrm{mg} / \mathrm{kg}, \mathrm{Mg}: 317 \mathrm{mg} /$ $\mathrm{kg}, \mathrm{Na}: 833 \mathrm{mg} / \mathrm{kg}, \mathrm{P}: 1752 \mathrm{mg} / \mathrm{kg})$ ef la $2 \mathrm{e}$ traite $(\mathrm{Zn}: 9 \mathrm{mg} / \mathrm{kg}, \mathrm{Ca}: 2089 \mathrm{mg} / \mathrm{kg}$, $\mathrm{Mg}: 209 \mathrm{mg} / \mathrm{kg}$, Na: $591 \mathrm{mg} / \mathrm{kg}, \mathrm{P}: 1409 \mathrm{mg} / \mathrm{kg})$. La diminution est plus lente ensuite jusqu'à la $5^{e}$ traite $(\mathrm{Zn}: 6 \mathrm{mg} / \mathrm{kg}, \mathrm{Ca}: 1806 \mathrm{mg} / \mathrm{kg}, \mathrm{Mg} 151 \mathrm{mg} / \mathrm{kg}$, $\mathrm{Na}: 508 \mathrm{mg} / \mathrm{kg}, \mathrm{P}: 1241 \mathrm{mg} / \mathrm{kg}$ (fig. 1).

La teneur en phosphatase alcaline suit la même évolution ; elle est élevée dans le lait de la première traite $\left(267 \mu \mathrm{mol} . \mathrm{mn}^{-1} \cdot \mathrm{kg}^{-1}\right)$; elle diminue de façon importante à la $2^{\mathrm{e}}$ traiłe $\left(116 \mu \mathrm{mol} . \mathrm{mn}^{-1} \cdot \mathrm{kg}^{-1}\right)$ puis plus lentement jusqu'en fin d'expérimentation ( $41 \mu \mathrm{mol} . \mathrm{mn}^{-1} \cdot \mathrm{kg}^{-1}$ à la $8 \mathrm{e}$ traite) (fig. 1).

L'influence du numéro de traite sur les teneurs en potassium n'esł pas significative. Toutefois, cette teneur augmente assez nettement entre la $1^{\text {re }}$ traite $(1321 \mathrm{mg} /$
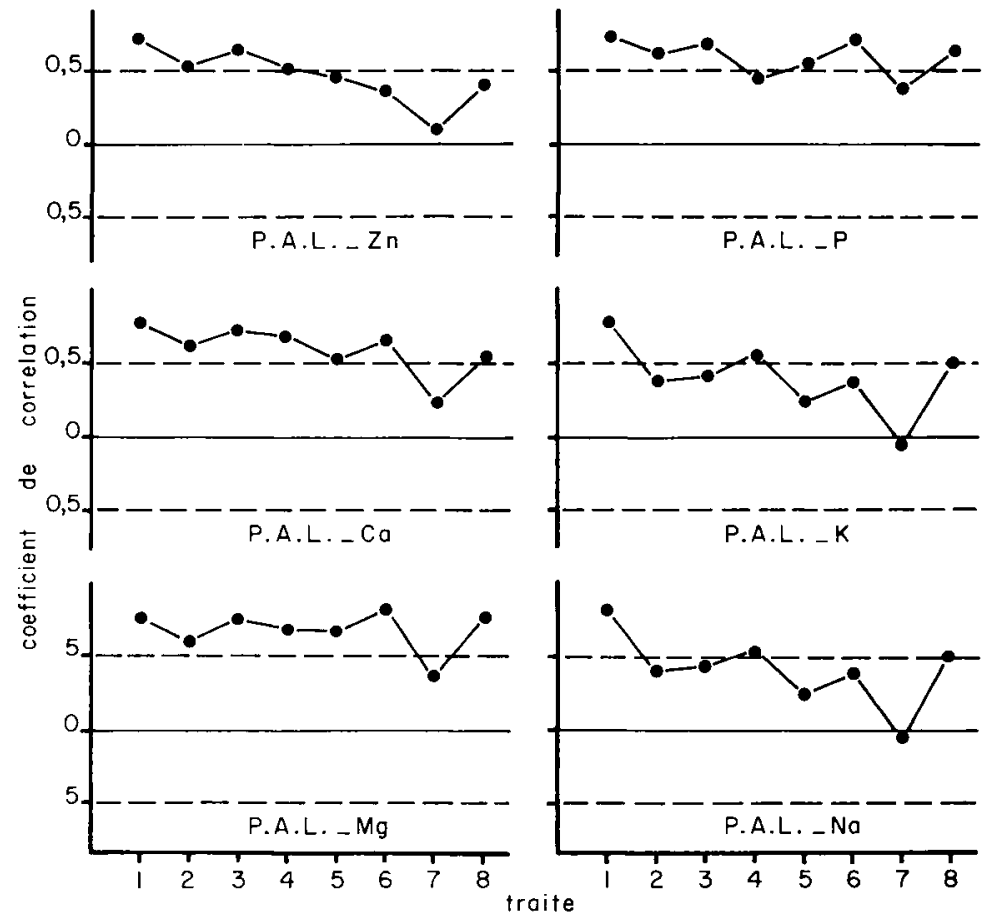

FIG. 2. - Evolution des coefficients de corrélation entre les quantités de phosphatase alcaline et les quantités de minéraux transportés dans le lait. 
$\mathrm{kg}$ ) et la $3^{\mathrm{e}}$ traite $(1565 \mathrm{mg} / \mathrm{kg})$. Elle se maintient ensuite entre 1430 ef $1520 \mathrm{mg} / \mathrm{kg}$ (fig. 1).

Par ailleurs, le test statistique de Fisher permet de montrer que ces résultats ne sont liés ni à la race, ni au régime alimentaire des animaux.

L'étude de l'évolution des coefficients de corrélation permet de mesurer l'importance des liaisons entre les quantités de phosphatase alcaline et les quantités d'éléments minéraux excrétés dans le lait (fig. 2). Entre les quantités de phosphatase alcaline et les quantités de potassium et sodium les liaisons sont faibles et variables. Par contre, entre les quantités de phosphatase alcaline ef les quantités de zinc, de calcium, de magnésium ef de phosphore les relations sont toujours positives et les coefficients de corrélation sont souvent élevés.

\section{Discussion.}

La sécrétion lactée étant de type apocrine, ces résultats permettent de suggérer une localisation très voisine de la phosphatase alcaline et des ions $\mathrm{Ca}, \mathrm{Mg}, \mathrm{Zn} \ldots$ Rappelons que le modèle de la structure physique de la membrane du globule gras rapporté par Peereboom (1970) comprend une couche externe de particules lipoprotéiques liée à la face interne de la membrane par des ions bivalents tels que $\mathrm{Ca}^{2+}$, $\mathrm{Mg}^{2+}$...

De plus, ces résultats permettent d'envisager une participation de la phosphatase alcaline du lait au transport, et peut-être d'une façon plus générale, au métabolisme de ces ions bivalents.

Dans un précédent travail (Linden ef al., 1977), nous avions déterminé que la constante de dissociation du complexe enzyme- $\mathrm{Mg}^{2+}$ est de $30 \mu \mathrm{M}$ à $\mathrm{pH} 8,5$ et que la constante de dissociation de $\mathrm{Zn}^{2+}$ du site $\mathrm{Mg}^{2+}$ est de $0,3 \mu \mathrm{M}$ à pH 8,5, $25^{\circ} \mathrm{C}$. Comme on le constate, le rapport de ces deux constantes d'affinité joue dans le même sens que celui des concentrations de ces ions dans le lait.

En outre, des recherches antérieures ont montré que l'enzyme du lait n'est sans doute pas impliqué dans des processus nécessitant la manifestation d'une activité monophosphoestérasique ou phosphoprotéine phosphatasique (Lorient ef Linden, 1976). Seule, l'hypothèse d'une participation de l'enzyme au transport de différentes substances telles que certains ions minéraux, paraît pour l'heure, vraisemblable. Ajoutons que les travaux d'histochimie de Girardie (1967) montrent précisément que le rôle de la phosphatase alcaline du lait dans la synthèse mammaire ne semble concerner que le transport de substances du milieu extérieur à travers les membranes.

Les phosphatases alcalines de mammifères connues sont incorporées à des membranes au travers desquelles ont lieu d'importants échanges de métabolites. Il paraît done vraisemblable de leur attribuer un rôle effectif dans le transpori des ions métalliques ou du phosphore. 


\section{Références}

ASCHAFFENBURG R., MULLEN J. E. C., 1948. A rapid and simple phosphatase test for milk. J. Dairy Res., 16, 58-67.

BAMMAN E., NOVOTNY E., ROHR L., 1948. Zur colorimetrischer Bestimmung der Phosphosaüre. Chem. Ber., 81, 438-449.

BELLANGER J., LAMAND M., 1975. Méthode de dosage du cuivre et du zinc plasmatique. Bull. CRZV Theix-INRA, no 20.

GIRARDIE J., 1967. Localisation optique et ultrastructurale de l'activité phosphatasique alcaline sans l'épithélium mammaire. C. R. Acad. Sci. Paris, Série D, 264, 2064-2067.

GUÉGUEN L., 1971. La composition minérale du lait et son adaptation aux besoins minéraux du jeune. Ann. Nutr. Alim., 25, A335-A381.

LINDEN G., GOHIER B., ALAIS C., TARODO de la FUENTE B., 1970. Essais de dessalification des produits laitiers par électrodialyse. Lait, 50, 511-523.

LINDEN G., ALAIS C., 1976. Phosphatase alcaline du lait de vache. II. Structure sous-unitaire, nature métalloprotéique ef paramètres cinétiques. Biochim. Biophys. Acta, 429, 205-213.

LINDEN G., CHAPPELET-TORDO D., LAZDUNSKI M., 1977. Milk alkaline phosphatase : stimulation by $\mathrm{Mg}^{2+}$ and properties of the $\mathrm{Mg}^{2+}$ site. Biochim. biophys. Acta, 483, 100-106.

LORIENT D., LINDEN G., 1976. Dephosphorylation of bovine casein by milk alkaline phosphatase. J. Dairy Res., 43, 19-26.

MARAVAL B., VIGNON B., 1977. Evolution de la composition minérale du lait de vache pendant la phase ascendante de la lactation. Différences entre les animaux primipares et les autres. Bull. E. N. S. A. I. A. Nancy, 19, 29-36.

PEEREBOOM J. W. C., 1970. Studies on Alkaline Milk phosphatase III : A theory for the mechanism of reactivation of alkaline milk phosphatase in a model system. Feffe Seifen Anstrichm. 72, 299-308. 